\title{
Problem-solving combined with ultrasonography: a strategy for internists to improve case management and reduce diagnostic errors
}

\author{
Daniela Tirotta, Vittorio Durante \\ UO Medicina di Cattolica, AUSL Romagna, Italy
}

\begin{abstract}
The rate of errors in the diagnostic process is reported in the literature to be between $10 \%$ and $15 \%$. Most of these errors are various forms of cognitive errors, which can be reduced by using a problem-solving approach. Here we present two cases showing how a problem-solving approach integrated with ultrasound information, often available to internists using point-of-care instruments, and clinical feed-back can help to reach the correct diagnosis and aid the management of complex cases of internal medicine.
\end{abstract}

\section{Introduction}

The rate of errors in the diagnostic process is reported in the literature to be between $10 \%$ and $15 \%$, with the rate being highest in specialties in which patients are diagnostically undifferentiated, such as emergency medicine, general practice and internal medicine. ${ }^{1}$ While defects or deficiencies in organizational boundaries, care processes and operators are the bases of error in the care of patients, errors in the diagnostic process can arise from various forms of cognitive errors, such as: $:^{2-4}$ i) context errors, in which the physician inappropriately limits consideration to only one set of diagnostic possibilities, in lieu of others; ii) availability errors, in which the physician chooses the most likely diagnosis over conditions that are rarer, or chooses conditions that he or she is most familiar with;

Correspondence: Daniela Tirotta, UO Medicina di Cattolica, AUSL Romagna, via Beethoven 1, 47841 Cattolica (RN), Italy.

Fax: +39.0541 .966290 .

E-mail: danitirotta@libero.it

Key words: abdominal ultrasonography, problem solving, diagnostic errors.

Conflict of interests: the authors declare that they have no potential conflict of interests.

Received for publication: 20 July 2013.

Revision received: 22 August 2013.

Accepted for publication: 2 September 2013.

This work is licensed under a Creative Commons Attribution NonCommercial 3.0 License (CC BY-NC 3.0).

CCopyright D. Tirotta and V. Durante, 2014

Licensee PAGEPress, Italy

Italian Journal of Medicine 2014; 8:11-18

doi:10.4081/itjm.2014.410 and iii) premature closure, such that once a plausible condition is identified, other possibilities are not fully considered - the physician just stops thinking.

Clinical problem-solving is based on hypotheses and deduction but also on a probabilistic approach. It is a sequential deductive process, because it involves the acquisition of data by searching and screening, interpretation of data, hypothesis generation and evaluation. During the course of clinical problem-solving physicians formulate hypotheses and analyze data to test the hypotheses. The approach is, however, also probabilistic because it is based on associations between clinical variables (such as frequency or prevalence) and provides a measure of likelihood that a hypothesis is correct. ${ }^{5}$

Most errors arise when only an intuitive approach is used; in contrast, conclusions drawn from an analytic assessment are generally reliable. Analytic failures can occur, but these are usually due to other factors such as cognitive overload, fatigue or emotional perturbations. ${ }^{1}$

In 2004, the American Institute of Ultrasound in Medicine concluded that the concept that ultrasound could be the stethoscope of the future had in fact already become reality and a recent review published in the New England Journal of Medicine highlighted the numerous applications of point-of-care ultrasonography in medicine (Table 1). ${ }^{6}$ Indeed, appropriate use of ultrasonography can reduce medical errors, provide a real-time diagnosis, replace or complement more complex imaging methods and, sometimes, reduce the need to consult radiologists for particularly demanding investigations. It should, however, be emphasized that the indiscriminate use of ultrasonography can lead to unnecessary interventions in the case of false positive findings and inappropriate interventions in the case of false negative ones.

The following two cases are focused on the usefulness of problem-solving, combined with internist ultrasonography to facilitate the diagnostic process, reducing diagnostic error. 


\section{Case \#1. Jaundice with a long unrecognized etiology}

\section{Description of the case}

A 60-year old woman was referred to us with severe jaundice, anorexia, and fever, associated with microcytic anemia (hemoglobin $8.1 \mathrm{~g} / \mathrm{dL}$, mean corpuscular volume $81 \mathrm{fL}$ ), neutrophilic leukocytosis (white blood cell count $28,900 / \mathrm{mm}^{3}$, neutrophils $25,020 / \mathrm{mm}^{3}$ ), biological inflammatory syndrome [Creactive protein (CRP) $178 \mathrm{mg} / \mathrm{L}$, erythrocyte sedimentation rate $120 \mathrm{mg} / \mathrm{L}$ ], abnormal liver function tests [aspartate aminotransferase (AST), $157 \mathrm{U} / \mathrm{L}$, alanine aminotransferase (ALT) $71 \mathrm{U} / \mathrm{L}$, total bilirubin $173 \mathrm{mg} / \mathrm{dL}$, direct bilirubin $9.3 \mathrm{mg} / \mathrm{dL}$, gammaglutamyltransferase (GGT) $107 \mathrm{U} / \mathrm{L}$, alkaline phosphatase (ALP) $364 \mathrm{U} / \mathrm{L}$ ] and abnormal coagulation tests (prothrombin time $1.56 \mathrm{~s}$ ). The patient had taken antibiotic therapy (ceftriaxone $1 \mathrm{~g}$ i.m.) for a week at home, without benefit.

On physical examination the patient had jaundice of the skin and mucosa, normal hemodynamic parameters (blood pressure 110/70 $\mathrm{mmHg}$, heart rate 100 beats $/ \mathrm{min}, \mathrm{SaO}_{2} 90 \%$ ), and a body temperature of $38^{\circ} \mathrm{C}$.

She had a history of neurological complications from previous meningitis and psychiatric manifestations. She had had multiple admissions to other medical wards for recurrent cholangitis, associated with intrahepatic cholestasis (no documentation of this was available, except for a few discharge letters). One month previously she had been diagnosed with bile duct cancer and a plastic biliary stent had been placed by endoscopic retrograde cholangiopancreatography (ERCP).

Abdominal computed tomography (CT) had been performed on that occasion, which showed solid tissue of the proximal common bile duct, below the confluence of the bile, with dilatation of the intrahepatic bile duct. Pericaval, peripancreatic, inter-aortocaval and hepatic hilar lymph nodes were enlarged. CT scan of the chest showed bilateral pulmonary nodules, compatible with metastases (maximum diameter of $1 \mathrm{~cm}$ ). ERCP showed lithiasis of the common bile duct (1.5 $\mathrm{cm})$ and stenosis $(2 \mathrm{~cm})$ of the common hepatic duct with dilatation of the intrahepatic bile duct.

The patient was not treated with chemotherapy (gemcitabine), in consideration of her psychiatric manifestations.

\section{Formulation of the clinical problem}

The clinical problem was jaundice, anorexia, and fever, associated with microcytic anemia, biological inflammatory syndrome, alterations of liver and coagulation tests in a patient with a biliary stent for cancer of the bile duct.

This clinical problem raises two questions: i) What are: the possible causes of extrahepatic cholestasis in adults? and ii) What are the potential complications in patients with cholangiocarcinoma and palliative stenting?

The possible causes of extrahepatic cholestasis in adults are i) lithiasis of the common bile duct; ii) neoplasms (cholangiocarcinoma, carcinoma of the ampulla); iii) pancreatic diseases (neoplasm of pancreatic head, chronic pancreatitis, pancreatic pseudocyst of the head); iv) stenosis of the common bile duct and congenital malformations (cysts, Caroli's disease); and v) extrahepatic cholestasis without pathological significance in the post-cholecystectomy setting and in elderly subjects. ${ }^{7}$

The potential complications in patients with cholangiocarcinoma and palliative stenting are: i) recurrent sepsis; ii) biliary obstruction and occlusion of the stent; iii) progression of the malignancy; and iv) acute cholecystitis.

\section{Formulation of the diagnostic hypotheses}

In the light of the clinical problem and its possible causes, we formulated three diagnostic hypotheses: i) a new episode of cholangitis associated with obstruction of the biliary stent; ii) a new episode of cholangitis secondary to displacement of the biliary stent; and iii) a super-infection of prosthetic material with secondary sepsis.

Table 1. Applications of point-of-care ultrasonography in some medical specialties.

\begin{tabular}{ll}
\hline Specialty & Ultrasound applications \\
\hline Cardiology & Echocardiography, intracardiac assessment \\
\hline Critical care medicine & Procedural guidance, pulmonary assessment, focused echocardiography \\
\hline Emergency medicine & FAST, focused emergency assessment, procedural guidance \\
\hline Endocrinology & Assessment of thyroid and parathyroid glands, procedural guidance \\
\hline Nephrology & Vascular access for dialysis \\
\hline Neurology & Transcranial Doppler, peripheral nerve evaluation \\
\hline Pulmonary medicine & Transthoracic pulmonary assessment, endobronchial assessment, procedural guidance \\
\hline Rheumatology & Monitoring of synovitis, procedural guidance \\
\hline Urology & Renal, bladder and prostate assessment, procedural assessment \\
\hline
\end{tabular}

Modified from Moore and Copel, $2011 .^{6}$ 


\section{Abdominal ultrasonography}

In order to obtain more information we performed an ultrasound of the abdomen (Figure 1).

This showed that the liver contained multiple echo-free lesions secondary to dilatation of the intrahepatic bile duct, dilatation of the bile duct with a block near the outlet in the papilla. There were no stones in the gallbladder.

We reviewed the case and asked ourselves two further questions: i) What is the meaning of the ultrasound evidence of cysts communicating with the intrahepatic bile duct? and ii) Had we studied the history sufficiently, before the diagnosis of cholangiocarcinoma, of the intrahepatic cholestasis and recurrent cholangitis in the absence of gallbladder lithiasis?

Considering the possible etiologies of multiple cysts in the liver, two groups can be distinguished. Those in which the cystic lesions communicate with the bile duct and those in which they do not. ${ }^{8}$

Cystic lesions that do not communicate with the bile duct include hepatorenal polycystosis, liver polycystosis and biliary hamartomas.

Hepatorenal polycystosis (PKD1 or PKD2) is one of the most common hereditary diseases. It is a monogenic, autosomal dominant condition with an incidence of 1:1-2000. Liver cysts are more numerous and voluminous in women and in older age renal involvement prevails. Ultrasonography is used in the diagnosis of complications, of which the most frequent are intracystic hemorrhage, infection and post-traumatic rupture.

Liver polycystosis is rare. It has been described in about 50 families. Its manifestations and complications are similar to those of hepatorenal polycystosis.

Biliary hamartomas (Von Meyenburg complexes) are embryogenic abnormalities. They may be associated with polycystic kidney disease, Caroli's disease, and congenital hepatic fibrosis. Rarely, they may undergo neoplastic transformation. Ultrasound findings are from two to multiple hypoechoic, hyperechoic or heterogeneous lesions. The diagnosis is made by nuclear magnetic resonance (NMR) imaging.

Cystic lesions that are in contact with the bile duct include Caroli's disease and syndrome, and peribiliary cysts.

Cystic dilations may be found in contact with the wall of the main extrahepatic bile duct and periportal veins (diameter from 1 to $2 \mathrm{~cm}$ ), predominantly on the left. They are described in severe liver disease (cirrhosis, portal hypertension) and may be associated with polycystic liver/kidney, infection, metastases, complications after transplantation, and localized compression of the bile duct. The hypothesized pathogeneses include congenital, ischemic and mechanical mechanisms. The diagnosis is made by NMR imaging.

The other question we asked when reviewing the case was, independently of the diagnosis of cholangiocarcinoma, what are the causes of recurrent cholangitis in the absence of gallbladder lithiasis? The possibilities include primary sclerosing cholangitis, Caroli's disease and recurrent pyogenic cholangitis.

\section{Additional diagnostic hypotheses in the light of the information acquired}

Applying a cognitive approach to problem-solving and integrating the information from ultrasound studies we had two elements: i) the detection of multiple cystic dilations communicating with the intrahepatic bile duct and ii) a history of recurrent cholangitis without gallbladder lithiasis in a patient with cholangiocarcinoma. These elements led us to suspect Caroli's disease complicated by cholangiocarcinoma, with current stenosis of the palliative stent and secondary cholangitis.

\section{Verification of the hypothesis}

Blood cultures were negative. We did not perform cholangio-NMR considering the psychiatric manifestations, but did carry out ERCP which revealed the intrahepatic bile duct with dilated cysts, in confirmation of the suspected Caroli's disease, and stenosis of the common bile duct.

\section{Management and clinical evolution}

Broad-spectrum antibiotics (piperacillin-tazobactam) were given. The stenosis of the common bile duct was relieved and a new metal stent was placed. This was well tolerated, the patient became afebrile and her laboratory parameters improved (total bilirubin 4.71 $\mathrm{mg} / \mathrm{dL}$, direct bilirubin $2.48 \mathrm{mg} / \mathrm{dL}$ ).

The patient was discharged with medium-dose steroid therapy, ursodeoxycholic acid $(10 \mathrm{mg} / \mathrm{kg})$ and the indication for abdominal ultrasound evaluation in first-degree relatives.

Two months later she had a new episode of cholangitis due to partial obstruction of the stent, with a partial response to antibiotic therapy. Seven months after the first hospitalization she was admitted again because of another episode of cholangitis, for which she was given antibiotics (full-dose piperacillin-tazobactam), hydrated and treated with steroids (prednisone $40 \mathrm{mg}$ ). Despite this management, the cholangitis evolved into sepsis and the patient died.

\section{Focus on Caroli's disease}

Caroli's disease is a rare congenital condition (incidence $1: 1,000,000$ with males and females equally

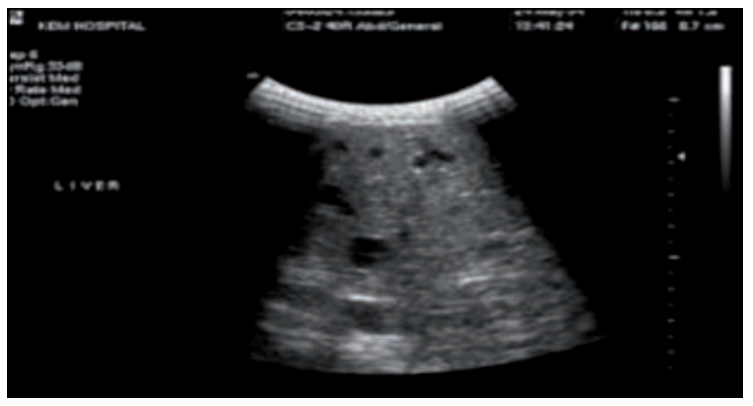

Figure 1. Abdominal ultrasound of case \#1. 
affected), characterized by fusiform or saccular dilatations of larger diameter intrahepatic bile ducts. The pathogenesis is unknown, but it is often an autosomal recessive trait, especially if associated with polycystic kidney disease.

The differential diagnoses include primary sclerosing cholangitis, polycystic liver disease (hepatic and renal cysts), recurrent pyogenic cholangitis (this is difficult to differentiate but there is sepsis and dilatation of extrahepatic as well as intrahepatic bile ducts with unusual saccular dilatations), and choledochal cysts (also hereditary, but cysts involve only the extrahepatic bile duct).

Patients begin to develop symptoms of Caroli's disease between 5-20 years old, or sometimes even later ( $80 \%$ of events occur in patients $<30$ years old). Following the onset of the first symptoms there is a sharp deterioration in the patient's quality of life, with the development of intrahepatic stones, intrahepatic abscesses, sepsis, which causes recurrent fever, jaundice, constant pain in the right upper quadrant of the abdomen, and acute cholangitis (64\%). Potential complications include abscess, intra- and extra-hepatic cholelithiasis, cholangiocarcinoma (2.5-16\%), cholangitis, liver cirrhosis and, rarely, amyloidosis.

The diagnosis is made on the basis of laboratory tests showing cholestasis, neutrophilic leukocytosis, vitamin $\mathrm{K}$ malabsorption and a reduction in hepatic synthetic function; demonstration of the continuity of the cystic lesions with the biliary tract by abdominal ultrasound, CT of the abdomen, ERCP (the gold standard), percutaneous transhepatic cholangiography or cholangio-NMR; and liver histology showing localized and non-obstructive dilatation of the bile ducts, intraluminal protrusions of the ductal walls and intraductal vascular tracts (arterial and venous portals).

An associated condition is Caroli's syndrome or congenital hepatic fibrosis. Whereas Caroli's disease involves the major tracts of bile ducts, the syndrome affects all parts of the intrahepatic ducts, small and large. Caroli's syndrome is frequently associated with polycystic kidney disease and can be inherited in an autosomal dominant or autosomal recessive manner; other associations are sponge kidney and tubular ectasia (Cacchi-Ricci's disease).

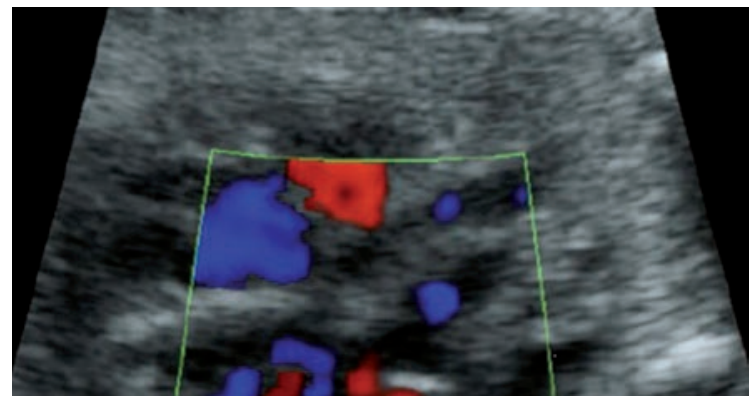

Figure 2. Abdominal ultrasound of case \#2 showing left intrahepatic cholestasis.
The hypothesized pathogenesis of Caroli's disease is that biliary stasis enables carcinogenic substances contained in the bile to provoke chronic inflammation and dysplasia of biliary epithelium.

The treatment depends on the extent of the anomalies. Localized forms can be managed with resection, lobectomy or hemi-hepatectomy, which often gives full and permanent benefit. Widespread forms are treated conservatively, with appropriate antibiotic therapy for cholangitis, ursodeoxycholic acid for intrahepatic lithiasis, endoscopic therapy (sphincterotomy for clearance of intrahepatic stones), internal bypass, and liver transplantation (although the prognosis is poor if there is concomitant liver fibrosis and cholangitis and is not recommended in the case of cholangiocarcinoma).

First-degree relatives should undergo abdominal ultrasound screening.

\section{Case \#2. Abdominal pain, vomiting and fever}

\section{Description of the case}

A 44-year old man came to our observation because of abdominal pain, vomiting and fever. He had a history of ulcerative colitis in clinical remission, which had been diagnosed a few years previously. In the preceding 5 years he had been seen occasionally in outpatient clinics and been admitted to hospital with a diagnosis of jaundice secondary to biliary lithiasis, which was managed with cholecystectomy and therapeutic ERCP. However, the persistence of cholestasis without jaundice led to the performance of a liver biopsy (non-diagnostic) about 1 year previously. The patient was not taking any medications on a chronic basis, except for non-steroidal anti-inflammatory drugs for occasional episodes of migraine.

Physical examination was normal, except for abdominal tenderness.

The laboratory findings were as follows: neutrophil leukocytosis (white blood cell count $15,000 / \mathrm{mm}^{3}$, neutrophils $\left.12,000 / \mathrm{mm}^{3}\right)$, biological inflammatory syndrome (CRP 91-27 mg/L), altered liver tests (ALT 371-65 U/L, ALP 485-280 U/L).

Abdominal ultrasonography revealed left intrahepatic cholestasis (Figure 2).

\section{Formulation of the clinical problem}

The clinical problem was jaundice and fever in a patient with persistent intrahepatic cholestasis and a previous diagnosis of chronic inflammatory bowel disease.

This clinical problem raises at least three questions: i) What is cholestasis without jaundice? ii) What are the causes of intrahepatic cholestasis in adults? and iii) What medications cause cholestatis?

Cholestasis without jaundice is often diagnosed following the incidental discovery of asymptomatic elevations of ALP and GGT. An increase in bilirubin levels is a late event. An isolated increase of GGT is not specific to cholestasis and this enzyme is often induced by alcohol or drugs. An isolated increase of ALP can be observed in rare diseases (e.g., progres- 
sive familial intrahepatic cholestasis I and II, deficiency of bile acid synthesis), bone diseases (e.g. Paget's disease) and pregnancy. The cut-off values of the laboratory parameters are the subject of debate: ALP $>1.5$ and GGT $>3$ times the upper limit of normal have been proposed.

Table 2. Causes of intrahepatic cholestasis in adulthood.

\section{Hepatocellular cholestasis}

Sepsis, endotoxemia-induced cholestasis

Cholestatic variety of viral hepatitis

Alcoholic or non-alcoholic steatohepatitis

Drug or parenteral nutrition-induced cholestasis

Genetic disorders (e.g. ACB4 deficiency, intrahepatic cholestasis of pregnancy, erythropoietic protoporphyria, benign recurrent intrahepatic cholestasis, progressive familial intrahepatic cholestasis)

Malignant infiltrating disorders (e.g. hematologic disease, metastatic cancer)

Benign infiltrating disorders (e.g. amyloidosis, sarcoidosis hepatitis and other granulomatoses, storage diseases)

Paraneoplastic syndromes (e.g. Hodgkin's disease, renal carcinoma)

Ductal plate malformations (e.g. congenital hepatic fibrosis)

Nodular regenerative hyperplasia

Vascular disorders (e.g. Budd-Chiari syndrome, veno-occlusive disease, congestive hepatopathy)

Cirrhosis (any cause)

Modified from European Association for the Study of the Liver, 2009. ${ }^{9}$
Intrahepatic cholestasis can be divided into hepatocellular and cholangiocellular forms. The causes of these two forms of intrahepatic cholestasis in adulthood are shown in Table 2. ${ }^{9}$

Medications that can cause cholestasis are listed in Table 3.

\section{Cholangiocellular cholestasis}

Primary biliary cirrhosis (AMA+/AMA-)

Primary sclerosing cholangitis

Overlap syndromes of primary biliary cirrhosis and primary sclerosing cholangitis with autoimmune hepatitis

\begin{tabular}{l}
\hline IgG4-associated cholangitis \\
\hline Idiopathic adulthood ductopenia \\
\hline Ductal plate malformations: biliary hamartoma, Caroli's syndrome \\
\hline Cystic fibrosis \\
\hline Drug-induced cholangiopathy \\
Graft-versus-host disease
\end{tabular}

Secondary sclerosing cholangitis (due to various forms of cholangiolithiasis, ischemic cholangiopathies: hereditary hemorrhagic teleangiectasia, polyarteritis nodosa and other forms of vasculitis), infectious cholangitis related to AIDS and other forms of immunodepression, etc.)

Table 3. Medications that can cause intrahepatic cholestasis.

\begin{tabular}{ll}
\hline Hepatocellular cholestasis & Ductular/ductal cholestasis \\
\hline Sex hormones & Allopurinol \\
\hline Carbamazepine & Amoxicillin-clavulanic acid \\
\hline Chlorpromazine & Azathioprine \\
\hline Amoxicillin-clavulanic acid & Barbiturates \\
\hline Trimethroprim-sulfamethoxazole & Captopril \\
\hline Erythromycin, clarithromycin & Carbamazepine \\
\hline Nitrofurantoin & Chlorpropamide \\
\hline Chlorpropamide & Clindamycin \\
\hline Azathioprine & Phenytoin \\
\hline Cyclosporine & Sulpiride \\
\hline Propafenone & Trimethoprim-Sulfamethoxazole \\
\hline Nifedipine & Medicinal herbs \\
\hline Medicinal herbs & \\
\hline NSAIDs, nimesulide & \\
\hline NSAIDs, non-steroidal anti-inflammatory drugs & \\
\hline
\end{tabular}

NSAIDs, non-steroidal anti-inflammatory drugs. 


\section{Formulation of the diagnostic hypotheses}

In the light of the clinical problem and the information available, we formulated four diagnostic hypotheses: i) cholestasis secondary to viral hepatitis, ii) primary biliary cirrhosis; iii) primary sclerosing cholangitis; and iv) infiltrative liver disease.

\section{Verification of the hypotheses}

Tests for hepatotropic viruses (hepatitis B surface antigen and core antigen, hepatitis $\mathrm{C}$ virus) were negative.

There was no evidence of organ-specific immune disorders (ANA, AMA, ASMA, LKM: negative) and immunoglobulins were within normal limits.

Cholangio-NMR showed moderate dilatation of the left intrahepatic hemi-biliary system, which had the appearance of a rosary and seemed to contain material with low-signal intensity, a finding compatible with sclerosing cholangitis. The presence of intra-ductal calculi could only be suspected; the right intra-hepatic biliary ducts, common bile duct, cystic duct and main pancreatic duct all appeared normal.

We made a diagnosis of sclerosing cholangitis

\section{Management and clinical evolution}

The patient was treated with ursodeoxycholic acid (15 mg/kg), obtaining progressive normalization of the indices of cholestasis and improvement of symptoms. At discharge, outpatient follow-up was recommended.

The patient did not return for further checks. However, 9 months later he had a new episode of abdominal pain associated with vomiting, which did not benefit from treatment with proton pump inhibitors. He underwent endoscopy which was positive for antral gastritis and a hiatal hernia. Because of the development of jaundice in the following days, he went to the Emergency Department of another hospital, where he was working, and where it was proposed that he was admitted. The patient refused and returned to our Unit.

On admission, the laboratory tests showed:

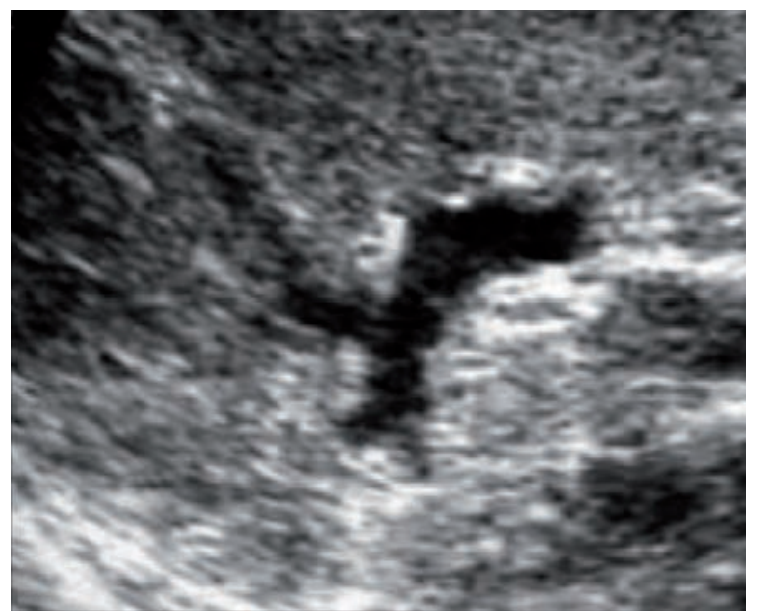

Figure 3. Abdominal ultrasound: unifocal stenosis. cholestasis (total bilirubin $7.16 \mathrm{mg} / \mathrm{dL}$, direct bilirubin $6.68 \mathrm{mg} / \mathrm{dL}$, ALP 489 U/L, GGT 423 U/L), hepatonecrosis (ALT $412 \mathrm{U} / \mathrm{L}$ ), and a biological inflammatory syndrome (CRP up to $113 \mathrm{mg} / \mathrm{L}$ ). Physical examination revealed cutaneous jaundice, a soft abdomen with pain, normal bowel sounds and hemodynamic parameters within the norm. Blood cultures were performed (negative) and the patient was started on empirical antibiotic therapy with piperacillin/tazobactam, which produced a progressive clinical remission.

The diagnosis was cholangitis in a patient with an acute exacerbation of primary sclerosing cholangitis and associated ulcerative colitis in remission. The patient was scheduled for outpatient follow-up of primary sclerosing cholangitis and ulcerative colitis.

\section{Focus on the internist ultrasound process in the case of detection of intrahepatic bile duct dilatation}

Intrahepatic bile duct abnormalities are often discovered by chance or, sometimes, during investigations for abdominal colic, jaundice (if multisegmental), or cholestasis. Ultrasound is the first step to differentiate the etiology of dilated intrahepatic bile ducts. The search for a neoplastic lesion associated with dilatation differentiates two groups of pathologies: biliary disease not caused by a parenchymal tumor and biliary disease secondary to a lesion in the adjacent parenchyma.

In the case of no abnormalities of the liver parenchyma, various patterns may be seen: unifocal stenosis, multifocal stenosis and no stenosis.

Unifocal stenosis (Figure 3) may develop as the outcome of surgery or biliary trauma and cause bile duct dilatation, or be the initial manifestation of primary sclerosing cholangitis (peripheral/segmental).

Multifocal stenosis (Figure 4) is typical of primary sclerosing cholangitis and autoimmune cholangitis. In the former case there is alternation of strictures and dilatations, giving the appearance of a string of pearls, and

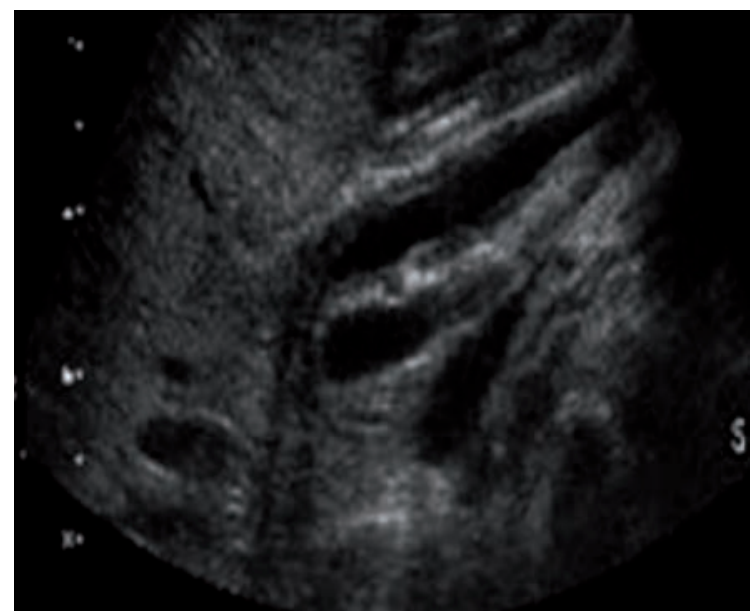

Figure 4. Abdominal ultrasound: multifocal stenosis. 
often intrahepatic lithiasis. The gold standard diagnostic investigation is cholangio-NMR, which shows thickening of the bile duct. The median age of affected patients is 40 years and $60-70 \%$ of cases occur in subjects with inflammatory bowel disease (especially ulcerative colitis). Markers of autoimmunity are negative, except ANCA, which is positive in $65-72 \%$ of cases. In autoimmune cholangitis there is extended segmental stenosis, frequently to the common hepatic duct, with dilatations before and after the strictures. Patients with this condition tend to be elderly subjects with autoimmune pancreatitis. They are ANCA-negative, sometimes have raised levels of IgG and respond to steroid therapy.

Finally, dilated intrahepatic bile ducts in the presence of normal liver parenchyma and absence of intrahepatic stenosis may be due to intrahepatic lithiasis (Figure 5), stricture of the biliary tree-digestive tract anastomosis, low phospholipid associated cholelithiasis (LPAC) syndrome, adenomas/papillomatosis, Caroli's disease and parasitosis (roundworm). Intrahepatic lithiasis is more frequent in the left side of the liver, is rare in Europe, and may occur in isolation or associated with gallstones and intrahepatic bile duct lithiasis. Rare, multiple adenomas of the biliary tract, which may be secretory (with secondary jaundice, abdominal pain) or not (31\%) degenerate into differentiated adenocarcinoma in $72 \%$ of cases, but resection is associated with a good prognosis.

The causes of unifocal or multifocal biliary dilatation associated with a mass (Figure 6) are usually biliary stricture due to malignancy, metastases, hepatocellular carcinoma infiltrating the bile ducts or cystic lesions. The malignant causes of biliary stricture include cholangiocarcinoma, intrahepatic cholangiocarcinoma and Klatskin's tumor. This tumor is poorly visualized on ultrasound if small, but is seen better in magnetic resonance imaging (contrast uptake after 3 $\mathrm{min})$. It is associated with intrahepatic lithiasis, sclerosing cholangitis or congenital abnormalities, with chronic inflammation of the bile duct. Cystic lesions include hydatid cysts, hepatic cystadenoma and simple cysts (in the case of hemorrhage).

\section{Recommendations for evaluating the adult patient with cholestasis}

The recommendations of the European Association for the Study of the Liver regarding evaluation of adults with cholestasis are listed below. The evidence and recommendations are graded according to the Grading of Recommendations Assessment Development and Evaluation (GRADE system) (Table 4). ${ }^{9}$

- A detailed history and physical examination are essential (III/C1).

- Abdominal ultrasonography is the first-line noninvasive imaging procedure in order to differentiated intra from extrahepatic cholestasis (III/C1).

- Testing for serum antimitochondrial antibodies (AMA) is mandatory in adults with chronic intrahepatic cholestasis (III/C1).

- Magnetic resonance cholangiopancreatography
(MRCP) is the next step to consider in patients with unexplained cholestasis (III/C1).

- Endoscopic ultrasound (EUS) is an alternative to MRCP for evaluation of distal biliary tract obstruction (II2/B1).

- Diagnostic endoscopic retrograde cholangiopancreatography (ERCP) should be reserved for highly selected cases. If the need for a therapeutic maneuver is not anticipated, MRCP or EUS should be preferred to ERCP because of the morbidity and mortality related to ERCP (II2/A1).

- A liver biopsy should be considered in patients with otherwise unexplained intrahepatic cholestasis and negative AMA test (II/C1).

Genetic testing for $A B C B 4$, when available, should be considered in patients with a negative AMA test and biopsy findings that might be compatible with primary biliary cirrhosis or primary sclerosing cholangitis.

\section{Discussion}

Both case descriptions show how analysis with problem-solving and feedback of the case are fundamental for reducing some cognitive errors (especially

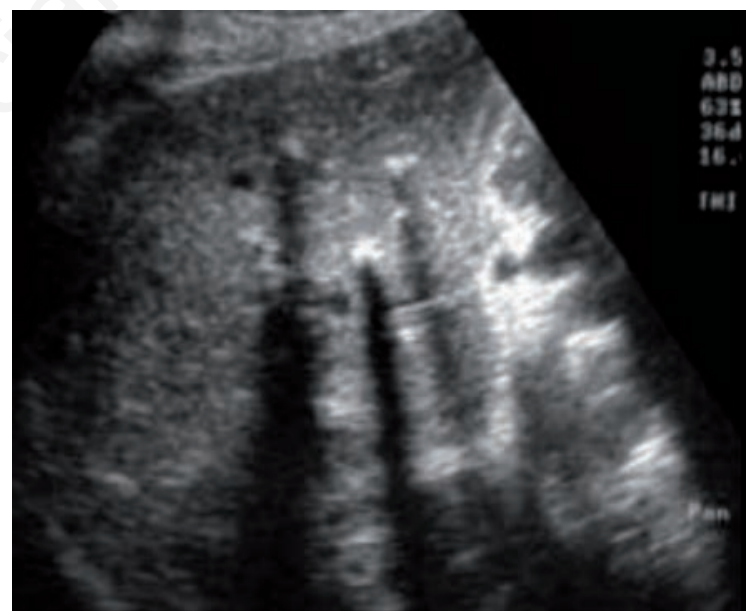

Figure 5. Abdominal ultrasound: intrahepatic lithiasis.

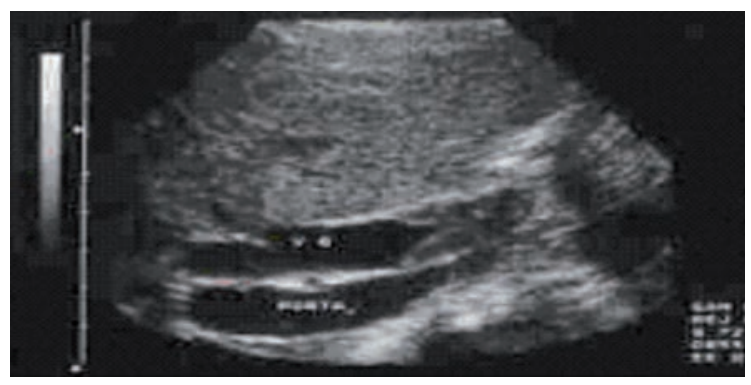

Figure 6. Abdominal ultrasound: unifocal/multifocal biliary dilatation associated with a mass. 
Table 4. Categories and grading of evidence and strength of recommendations.

\begin{tabular}{|c|c|c|}
\hline \multicolumn{3}{|c|}{ Categories of evidence } \\
\hline Grade & Evidence & \\
\hline $\mathrm{I}$ & Randomized controlled trials & \\
\hline II-1 & Controlled trials without randomization & \\
\hline II-2 & Cohort of case-control analytic studies & \\
\hline II-3 & Multiple time series, dramatic uncontrolled experiments & \\
\hline III & Opinions of respected authorities, descriptive epidemiology & \\
\hline \multicolumn{3}{|l|}{ Evidence grading } \\
\hline Evidence & Notes & \\
\hline High quality & Further research is very unlikely to change our confidence in the estimate of effect & A \\
\hline Moderate quality & $\begin{array}{l}\text { Further research is likely to have an important impact on our confidence in the estimate of effect } \\
\text { and may change the estimate }\end{array}$ & B \\
\hline Low quality & $\begin{array}{l}\text { Further research is very likely to have an important impact on our confidence in the estimate of effect } \\
\text { and is likely to change the estimate, any change of estimate is uncertain }\end{array}$ & $\mathrm{C}$ \\
\hline \multicolumn{3}{|c|}{ Recommendations } \\
\hline Strong & $\begin{array}{l}\text { Factors influencing the strength of recommendation included the quality of the evidence, } \\
\text { presumed patient important outcomes, and cost }\end{array}$ & 1 \\
\hline Weak & $\begin{array}{l}\text { Variability in preferences and values, or more uncertainty. Recommendation is made with less certainty, } \\
\text { higher cost or resource consumption }\end{array}$ & 2 \\
\hline
\end{tabular}

Modified from European Association for the Study of the Liver, 2009. ${ }^{9}$

context and availability errors) and picking up overlooked elements (e.g. the association between inflammatory bowel disease - primary sclerosing cholangitis; intrahepatic cholestasis without lithiasis - recurrent cholangitis).

Abdominal ultrasound by internists, combined with problem-solving, should be considered part of the case management of patients with cholestasis, with the aim of achieving two main outcomes: accelerating the diagnosis and reducing costs.

With regards to accelerating the diagnosis, the reduction in the diagnostic latency should be associated with a secondary reduction of mortality and improved quality of life of the patients. Indeed, in both of the cases presented here, the lack of an initial analytical approach (reasoning process with problem-solving combined with abdominal ultrasound) resulted in a late diagnosis. In the first case this determined the patient's death. However, the combined approach has, at least, made it possible to screen first-degree relatives for Caroli's disease. In the second case the diagnosis enabled the correct treatment to be given and appropriate follow-up to be planned (essential for the screening of cholangiocarcinoma and intestinal neoplasia).

With regards to a reduction of costs, although this is probable, there is a lack of cost-efficacy and costbenefit studies comparing ultrasound by internists and ultrasound by radiologists. In a search of PubMed we found only one cost-effectiveness study on the usefulness of contrast-enhanced ultrasonography in patients with hepatic lesions. ${ }^{10}$

\section{References}

1. Croskerry P. From mindless to mindful practice - cognitive bias and clinical decision making. $\mathrm{N}$ Engl J Med 2013;368:2445-8.

2. Ely JW, Graber ML, Croskerry P. Checklists to reduce diagnostic errors. Acad Med 2011;86:307-13.

3. Elstein AS, Schwarz A. Clinical problem solving and diagnostic decision making: selective review of the cognitive literature. BMJ 2002;324:729-32.

4. Agency for Healthcare Research and Quality. Diagnostic errors in medicine: what do doctors and umpires have in common? Rockville, MD: AHRQ; 2007. Available from: http:/www.webmm.ahrq.gov

5. Hardin LE. Research in medical problem solving: a review. J Vet Med Educ 2003;30:230-5.

6. Moore CL, Copel JA. Point-of-care ultrasonography. N Engl J Med 2011;364:749-57.

7. Rogoveanu I, Gheonea DI, Saftoiu A, Ciurea T. The role of imaging methods in identifying the causes of extrahepatic cholestasis. J Gastrointestin Liver Dis 2006; 15 : 265-71.

8. Vullierme MP, Vilgrain V. [Isolated or multifocal segmental intrahepatic bile duct dilatation]. J Radiol 2006; 87:500-12. [Article in French].

9. European Association for the Study of the Liver. EASL clinical practice guidelines: management of cholestatic liver diseases. J Hepatol 2009;51:237-67.

10. Piscaglia F, Leoni S, Cabibbo G, et al. Cost analysis of recall strategies for non-invasive diagnosis of small hepatocellular carcinoma. Dig Liver Dis 2010;42:729-34. 